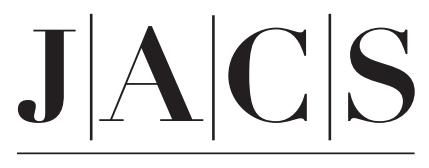

A R T I C L E S

Published on Web 06/12/2002

\title{
Stereochemical Effects in Supramolecular Self-Assembly at Surfaces: 1-D versus 2-D Enantiomorphic Ordering for PVBA and PEBA on $\mathrm{Ag}(111)$
}

\author{
Johannes V. Barth, ${ }^{* \dagger}$ Jens Weckesser, ${ }^{\dagger, \ddagger}$ Giancarlo Trimarchi,§ Masha Vladimirova," \\ Alessandro De Vita, $,{ }^{\S}, \|$ Chengzhi Cai, ${ }^{\perp, \#}$ Harald Brune, ${ }^{\dagger}$ Peter Günter,${ }^{\perp}$ and \\ Klaus Kern ${ }^{\dagger} \neq$ \\ Contribution from the Institut de Physique des Nanostructures, Ecole Polytechnique Fédérale de \\ Lausanne, CH-1015 Lausanne, Switzerland; Max-Planck-Institut für Festkörperforschung, \\ Heisenbergstrasse 1, D-70569 Stuttgart, Germany; INFM and Dipartimento di Ingegneria dei \\ Materiali, Università di Trieste, via A. Valerio 2, I-34127 Trieste, Italy; Institut Romand de \\ Recherche Numérique en Physique des Matériaux, CH-1015 Lausanne, Switzerland; and \\ Nonlinear Optics Laboratory, Eidgenössische Technische Hochschule Zürich, ETH-Hönggerberg, \\ CH-8093 Zürich, Switzerland
}

Received January 23, 2002

\begin{abstract}
We present investigations on noncovalent bonding and supramolecular self-assembly of two related molecular building blocks at a noble metal surface: 4-[trans-2-(pyrid-4-yl-vinyl)]benzoic acid (PVBA) and 4-[(pyrid-4-yl-ethynyl)]benzoic acid (PEBA). These rigid, rodlike molecules comprising the same complementary moieties for hydrogen bond formation are comparable in shape and size. For PVBA, the ethenylene moiety accounts for two-dimensional (2-D) chirality upon confinement to a surface; PEBA is linear and thus 2-D achiral. Molecular films were deposited on a $\mathrm{Ag}(111)$ surface by organic molecular beam epitaxy and characterized by scanning tunneling microscopy. At low temperatures (around $150 \mathrm{~K}$ ), both species form irregular networks of flat lying molecules linked via their endgroups in a diffusion-limited aggregation process. In the absence of kinetic limitations (adsorption or annealing at room temperature), hydrogen-bonded supramolecular assemblies form which are markedly different. With PVBA, enantiomorphic twin chains in two mirror-symmetric species running along a high-symmetry direction of the substrate lattice form by diastereoselective self-assembly of one enantiomer. The chirality signature is strictly correlated between neighboring twin chains. Enantiopure one-dimensional (1-D) supramolecular nanogratings with tunable periodicity evolve at intermediate coverages, reflecting chiral resolution in micrometer domains. In contrast, PEBA assembles in 2-D hydrogen-bonded islands, which are enantiomorphic because of the orientation of the supramolecular arrangements along low-symmetry directions of the substrate. Thus, for PVBA, chiral molecules form 1-D enantiomorphic supramolecular structures because of mesoscopic resolution of a 2-D chiral species, whereas with PEBA, the packing of an achiral species causes 2-D enantiomorphic arrangements. Model simulations of supramolecular ordering provide a deeper understanding of the stability of these systems.
\end{abstract}

\section{Introduction}

Chiral surfaces and films are of fundamental interest in enantioselective heterogeneous catalysis and in the study of enantiomeric selectivity in optical activity or electron-molecule interactions. ${ }^{1-9}$ Recent investigations employing the scanning tunneling microscope revealed that this issue can be addressed

\footnotetext{
* To whom correspondence should be addressed. E-mail: johannes.barth@epfl.ch.

† Ecole Polytechnique Fédérale de Lausanne.

$\doteqdot$ Max-Planck-Institut für Festkörperforschung.

$\S$ Università di Trieste and INFM, DEMOCRITOS Center for Research in Atomistic Simulations.

"Institut Romand de Recherche Numérique en Physique des Matériaux.

${ }^{\perp}$ Eidgenössische Technische Hochschule Zurich.

\# Present address: Department of Chemistry, University of Houston, Houston, TX 77204-5003.

(1) Jannes, G., Dubois, V., Eds. Chiral Reactions in Heterogeneous Catalysis; Plenum: New York, 1995.
}

successfully at the single molecule level with adsorbed species at solid surfaces. There are several aspects to chirality at surfaces. The simplest case concerns the adsorption of a molecule, which exhibits chirality in three dimensions (3-D).

(2) Baiker, A.; Blaser, H. U. In Handbook of Heterogeneous Catalysis; Ertl, G., Knözinger, H., Weitkamp, J., Eds.; VCH: Weinheim, 1997; Vol. 5, pp $2422-2430$.

(3) Lorenzo, M. O.; Baddeley, C. J.; Muryn, C.; Raval, R. Nature 2000, 404, $376-379$

(4) Byers, J. D.; Yee, H. I.; Petralli-Mallow, Z.; Hicks, J. M. Phys. Rev. B 1994, 49, 14643-14647.

(5) Hecht, L.; Barron, L. Chem. Phys. Lett. 1994, 225, 525.

(6) Maki, J. J.; Kauranen, M.; Persoons, A. Phys. Rev. B 1995, 51, 14251434.

(7) Verbiest, T.; Elshocht, S. V.; Kauranen, M.; Hellemans, L.; Snauwaert, J.; Nuckolls, C.; Katz, T. J.; Persoons, A. Science 1998, 282, 913-915.

(8) Ray, K.; Ananthavel, S. P.; Waldeck, D. H.; Naaman, R. Science 1999 $283,814-816$

(9) Fasel, R.; Cossy, A.; Ernst, K.-H.; Baumberger, F.; Greber, T.; Osterwalder, J. J. Chem. Phys. 2001, 115, 1020-1027. 
In favorable cases, the chirality of the individual adsorbed molecule can be directly determined. ${ }^{10,11}$ Moreover, for simple geometrical reasons, 3-D achiral molecules may become chiral upon adsorption because of the reduced symmetry at the surface; that is, they are two-dimensional (2-D) chiral. Beyond the individual molecule, molecular arrangements and films may be chiral or enantiomorphic. This includes, in particular, the possibility that chiral layers are formed from achiral, racemic, and chiral species. ${ }^{12-15}$ In some cases, indications of spontaneous chiral segregation of racemic films have been reported. ${ }^{16-19}$ When the substrate atomic order is affected, even chiral restructuring may occur. ${ }^{20}$ Finally, chirality may be expressed at the supramolecular level in discrete molecular assemblies at surfaces which are stabilized by noncovalent bonds. ${ }^{21-24}$

The understanding of chiral ordering at surfaces is in its infancy. The objective of the present investigations is to elucidate stereochemical effects in the chiral supramolecular assembly of organic molecules at surfaces. Specifically, we employed the two molecular building blocks 4-[trans-2-(pyrid4-yl-vinyl)]benzoic acid (PVBA) and 4-[(pyrid-4-yl-ethynyl)]benzoic acid (PEBA) designed for nonlinear optics applications. ${ }^{25-28}$ The structure formulas given in Figure 1 show that the two molecules are similar. Both species are rigid and planar. The identical endgroups, that is, a carboxylic acid ("head") and a pyridyl moiety ("tail"), are designed to form head-to-tail hydrogen bonds, a feature which is found in PVBA and PEBA powder samples. ${ }^{25-28}$ The ethenylene bridge of PVBA is substituted by an ethynylene bridge in PEBA leading to a slightly increased length of PEBA with respect to that of PVBA (for free molecules, the projection of the distance of the extremal $\mathrm{N}$ and $\mathrm{H}$ atoms on the symmetry axis of the pyridile moiety is 12.8 vs $12.4 \AA$; the distances between these two atoms are 12.8

(10) Lopinski, G. P.; Moffat, D. J.; Wayner, D. D. M.; Wolkow, R. A. Nature 1998, 392, 909-911.

(11) Lopinski, G. P.; Moffat, D. J.; Wayner, D. D. M.; Zgierski, M. R.; Wolkow, R. A. J. Am. Chem. Soc. 1999, 121, 4532-4533.

(12) Viswanathan, R.; Zasadzinski, J. A.; Schwartz, D. K. Nature 1994, 368 $440-443$.

(13) Sowerby, S. J.; Heckl, W. M.; Petersen, G. B. J. Mol. Evol. 1996, 43, 419-424.

(14) Walba, D. M.; Stevens, F.; Clark, N. A.; Parks, D. C. Acc. Chem. Res. 1996, 29, 591-597.

(15) Charra, F.; Cousty, J. Phys. Rev. Lett. 1998, 80, 1682-1685.

(16) Eckhardt, C. J.; Peachey, N. M.; Swanson, D. R.; Takacs, J. M.; Khan, M. A.; Gong, X.; Kim, J.-H.; Wang, J.; Uphaus, R. A. Nature 1993, 362, 614616.

(17) Nassoy, P.; Goldmann, M.; Bouloussa, O.; Rondalez, F. Phys. Rev. Lett. 1995, 75, 457-460.

(18) Stevens, F.; Dyer, D. J.; Walba, D. M. Angew. Chem., Int. Ed. Engl. 1996, 35, 900-901.

(19) Fang, H.; Giancarlo, L. C.; Flynn, G. W. J. Phys. Chem. B 1998, 102, $7311-7315$

(20) Schunack, M.; Lægsgaard, E.; Steensgard, L.; Johannsen, I.; Besenbacher, F. Angew. Chem., Int. Ed. 2001, 40, 2623-2626.

(21) Böhringer, M.; Morgenstern, K.; Schneider, W.-D.; Berndt, R. Angew. Chem., Int. Ed. 1999, 38, 821-823.

(22) Böhringer, M.; Schneider, W.-D.; Berndt, R. Angew. Chem., Int. Ed. 2000 39, 792-795.

(23) Barth, J. V.; Weckesser, J.; Cai, C.; Günter, P.; Bürgi, L.; Jeandupeux, O.; Kern, K. Angew. Chem., Int. Ed. 2000, 39, 1230-1234.

(24) Weckesser, J.; Vita, A. D.; Barth, J. V.; Cai, C.; Kern, K. Phys. Rev. Lett. 2001, 87, 096101.

(25) Cai, C.; Bösch, M.; Tao, Y.; Müller, B.; Gan, Z.; Kündig, A.; Bosshard, C.; Liakatas, I.; Jäger, M.; Günter, P. J. Am. Chem. Soc. 1998, 120, 8563.

(26) Cai, C.; Bösch, M.; Müller, B.; Tao, Y.; Kündig, A.; Bosshard, C.; Gan, Z.; Biaggio, I.; Liakatas, I.; Jäger, M.; Schwer, H.; Günter, P. Adv. Mater. 1999, 11, 745-749.

(27) Cai, C.; Müller, B.; Weckesser, J.; Barth, J. V.; Tao, Y.; Bösch, M. M.; Kündig, A.; Bosshard, C.; Biaggio, I.; Günter, P. Adv. Mater. 1999, 11, $750-754$

(28) Cai, C.; Bösch, M. M.; Bosshard, C.; Müller, B.; Tao, Y.; Kündig, A.; Kiy, M.; Biaggio, I.; Liakatas, I.; Günter, P.; Weckesser, J.; Barth, J. V.; Bürgi, L.; Jeandupeux, O.; Kern, K. In Anisotropic Materials - Approaches to Polar Order; Glaser, R., Kaszynski, P., Eds.; ACS Symposium Series; American Chemical Society: Washington, DC, 2001; pp 34-49. and $12.5 \AA$, respectively). Upon confinement to two dimensions, the angled molecular structure of PVBA imposes a chirality not present in the gas phase. Thus, two enantiomers exist which are labeled $\lambda$ and $\delta$ PVBA, respectively (cf. Figure 1a). By contrast, PEBA is linear, and thus the adsorbed molecule remains achiral at a surface (cf. Figure 1b). With both molecules, we disregard the asymmetry of the carboxylic acid group, which can rotate in the gas phase, as the hydrogen atom is expected to freely transport from one oxygen atom to the other at the surface, similar to related systems. ${ }^{29}$ The comparative study of these two molecules allows us to gain insight into the principles of hydrogen-bond formation and supramolecular self-assembly at metal surfaces; in particular, it contributes to the elucidation of the effects of shape and symmetry, that is, stereochemical aspects.

\section{Experimental Section}

2.1. Scanning Tunneling Microscopy. The experiments were performed with a home-built low-temperature STM $(5$ or $77 \mathrm{~K}$ operation), incorporated in a ultrahigh vacuum (UHV) chamber with a base pressure in the lower $10^{-11}$ mbar range. ${ }^{30,31}$ The $\mathrm{Ag}(111)$ crystal was prepared by cycles of argon ion sputtering $\left(700 \mathrm{eV}, 0.5 \mu \mathrm{A} / \mathrm{cm}^{2}\right)$ and subsequent annealing $(870 \mathrm{~K})$ resulting in large defect-free terraces of typically $1000 \AA$ width. PVBA and PEBA were deposited by organic molecular beam epitaxy using a conventional Knudsen cell at background pressures of $\sim 5 \times 10^{-10}$ mbar at sample temperatures in the range $100-300 \mathrm{~K}$. The employed deposition rate was $\sim 5 \times 10^{-4} \mathrm{ML} / \mathrm{s}$ as calibrated by STM (coverage given in terms of the saturated physical monolayer $\left(\Theta_{\mathrm{ph}}=1 \mathrm{ML}\right)$, where the ratio of adsorbed molecules per $\mathrm{Ag}$ surface atom is approximately 1:14 for both species). After preparation, the samples are transferred into the low-temperature STM freezing the obtained situation. All data presented here have been obtained after cooling the sample to $77 \mathrm{~K}$.

2.2. Model Simulations. (i) First-principles calculations and (ii) classical molecular dynamics (MD) techniques based on an empirical potential derived from first-principles results were performed. Firstprinciples calculations employ density functional theory (DFT) within the generalized gradient corrected approximation (GGA). In particular, we use the PW91 prescription to describe the electronic exchange and correlation effects. ${ }^{32}$ The Kohn-Sham electronic orbitals are expanded on a plane wave basis set up to a kinetic energy cutoff of $50 \mathrm{Ry}$. The interaction between core ions and valence electrons is represented by norm-conserving pseudopotentials of the Troullier-Martins form. ${ }^{33}$ Structural optimizations are carried out by damped dynamics for both the electronic and the ionic degrees of freedom, using the CarParrinello method. ${ }^{34}$ As a convergence criterion for structural relaxation, we require that the residual forces acting on all atoms be less than $5 \times$ $10^{-4}$ au (or about $0.03 \mathrm{eV} / \AA$ ).

Fully first-principles dynamical simulations of large supported supramolecular assemblies such as the ones studied here are not feasible with the currently available computing means. However, it is possible to model the intermolecular interactions with a classical force field in which the main electrostatic interaction is accounted for by partial Coulomb charges located on atoms and fitted to best reproduce the ab initio electrostatic potential of an isolated molecule..$^{24,35}$ This force field

(29) Hydrogen Transfer: Experiment and Theory. Limbach, H.-H., Manz, J., Eds. Ber. Bunsen-Ges. Phys. Chem. 1998, 102, 289-586.

(30) Hirstein, A. Ph.D. Thesis, EPF Lausanne, 1998

(31) Jeandupeux, O.; Bürgi, L.; Hirstein, A.; Brune, H.; Kern, K. Phys. Rev. B 1999, 59, 15926-15934.

(32) Perdew, J. P.; Wang, Y. Phys. Rev. B 1992, 45, 13244.

(33) Troullier, N.; Martins, J. L. Phys. Rev. B 1991, 43, 1993.

(34) Car, R.; Parrinello, M. Phys. Rev. Lett. 1985, 55, 2471.

(35) Vladimirova, M.; Stengel, M.; Vita, A. D.; Baldereschi, A.; Böhringer, M.; Morgenstern, K.; Berndt, R.; Schneider, W.-D. Europhys. Lett. 2001, $56,254$. 
a) 4-[trans-2-(pyrid-4-yl-vinyl)] benzoic acid

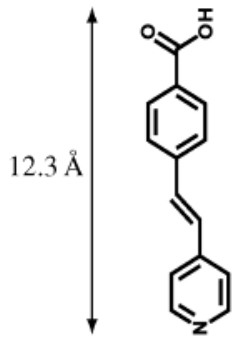

$\lambda$-PVBA

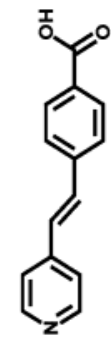

$\delta$-PVBA
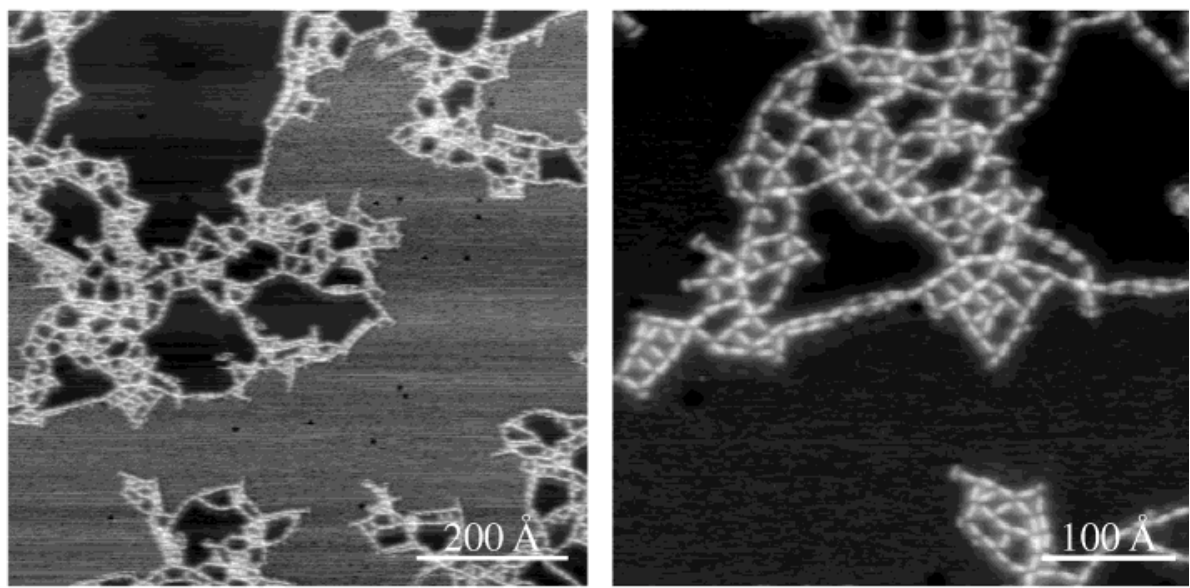

b) 4-[(pyrid-4-yl-ethynyl)] benzoic acid

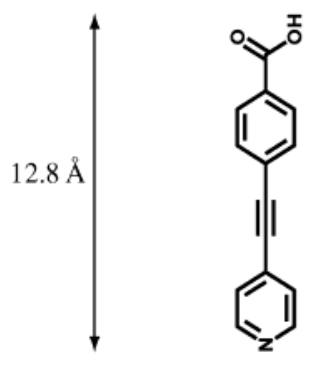

PEBA


Figure 1. Chemical structure formulas for the two related molecular species and STM topographs for low-temperature aggregation on Ag(111). (a) PVBA with the 2-D chiral species labeled $\lambda$ and $\delta$; (b) PEBA. With PVBA upon evaporation on $\operatorname{Ag}(111)$ at $125 \mathrm{~K}$, networks of meandering chains are formed demonstrating surface mobility at low temperature and attractive intermolecular interactions. For PEBA on $\operatorname{Ag}(111)$ evaporated at $T_{\mathrm{Ag}}=150 \mathrm{~K}$, networks are formed, which are more compact as in the case of PVBA. They either decorate step edges or form islands in flat terraces. The close-up views reveal that in both cases the flat-lying molecules interact predominantly via their endgroups, which is in line with the expected hydrogen bond formation. The growth scenarios can be considered as diffusion-limited aggregation of rodlike particles subject to anisotropic interactions.

has been shown in earlier work to reproduce in sufficient detail the features of assembled structures of the same class as those considered here. ${ }^{24,36}$ Simulated annealing classical MD simulations based on this force field have been carried out to identify the minimum energy configurations of a number of PVBA and PEBA supramolecular assemblies.

\section{Scanning Tunneling Microscopy Observations}

3.1. Diffusion-Limited Aggregation at Low Temperatures. STM topographs showing the situation evolving upon PVBA adsorption with the crystal held at $125 \mathrm{~K}$ are shown in Figure 1a. They reveal that PVBA is mobile on the $\operatorname{Ag}(111)$ surface at low temperatures and that networks of meandering chains are formed. No isolated molecules can be imaged on flat terraces. However, the noisy background and height difference $(\sim 0.2 \AA)$ on empty areas might be because of mobile molecules dragged by the STM tip. In the close-up view, the rodlike molecular shape is clearly discernible revealing that PVBA adsorbs flat on the $\operatorname{Ag}(111)$ surface. This is associated with $\pi$-bonding orbitals pointing toward the substrate and was similarly found for PVBA on the Pd(110) surface, ${ }^{37,38}$ and other large organic molecules with extended $\pi$-systems, for example,

(36) Böhringer, M.; Morgenstern, K.; Schneider, W.-D.; Berndt, R.; Mauri, F.; Vita, A. D.; Car, R. Phys. Rev. Lett. 1999, 83, 324-327. oligothiophenes on $\operatorname{Ag}(111) .{ }^{39,40}$ Attractive interactions between the molecular endgroups are operative, consistent with the directional interactions expected from hydrogen bond formation. Up to five endgroups come together in one junction. Typically, however, molecular strings evolve at the surface. Their smoothly curved shape involves PVBA molecules adsorbed at arbitrary angles with respect to the substrate lattice signaling that the substrate corrugation experienced by the molecules is rather weak in comparison with the lateral intermolecular bonding. This is attributed to the smoothness of the close-packed substrate geometry and the weak chemical bonding between the adsorbates and the noble metal surface. The formation of strings demonstrates that head-to-tail bonding between the molecules is preferred to a possible head-to-head coupling of two benzoic acid groups (in the latter case, exclusively, pairs of molecules would be formed). The observed growth scenario can be rationalized in terms of diffusion-limited aggregation of rodlike

(37) Weckesser, J.; Barth, J. V.; Kern, K. J. Chem. Phys. 1999, 110, 53515354

(38) Weckesser, J.; Barth, J. V.; Cai, C.; Müller, B.; Kern, K. Surf. Sci. 1999, $431,268-173$.

(39) Umbach, E.; Sokolowski, M.; Fink, R. Appl. Phys. A 1996, 63, 565-576.

(40) Soukopp, A.; Glöckler, K.; Kraft, P.; Schmitt, S.; Sokolowski, M.; Umbach, E.; Mena-Osteritz, E.; Bäuerle, P.; Hädicke, E. Phys. Rev. B 1998, 58, $13882-13894$. 

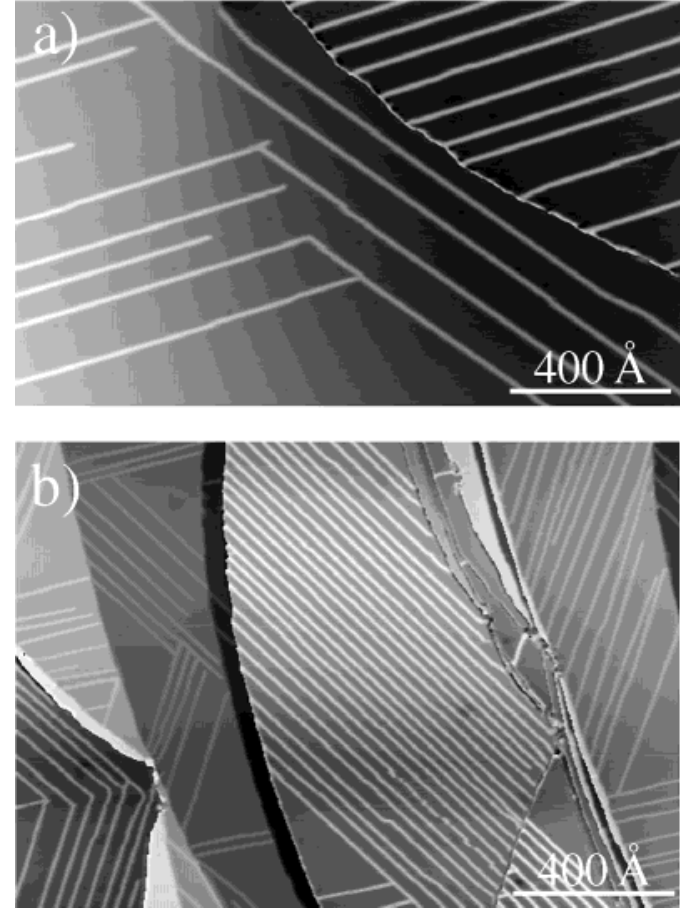

Figure 2. Stripe arrangements at small PVBA concentrations upon adsorption or annealing at $300 \mathrm{~K}$. (a) Albeit the mutual distances are about $200 \AA$ A, stripes are oriented parallel. The same grayscale range is used on both terraces, and the atomic step thus appears as a white line in the upper right of the STM image $\left(\Theta_{\mathrm{ph}}=0.1 \mathrm{ML}\right)$. (b) The three possible rotational domains of the stripe pattern reflect the 3-fold symmetry of the fcc(111) substrate (neighboring levels are represented with different gray scales). Small terraces are frequently free of adsorbates $\left(\Theta_{\mathrm{ph}}=0.2 \mathrm{ML}\right)$.

particles subject to anisotropic interactions. Accordingly, the irregularity of the formed agglomerates demonstrates that their shape is because of kinetic limitations, whereby thermal equilibrium is not attained.

In Figure 1b, STM data indicating a related aggregation mechanism for PEBA on $\mathrm{Ag}(111)$ (following deposition at 150 $\mathrm{K})$. On a large scale, irregular molecular networks as in the case of PVBA are formed, either decorating step edges or appearing as individual islands on flat terraces. Similar to the case of PVBA, the detail image shows that the molecules adsorb in a flat geometry and are interconnected via their endgroups. Again, the planar adsorption reflects the directed $\pi$-bonding of PEBA to the metal surface, and the interaction of molecular endgroups is associated with hydrogen bond formation. However, the PEBA network is more compact when compared to the one made from PVBA. This is attributed to the different molecular shape and is thus a steric effect.

3.2. One-Dimensional Hydrogen-Bonded Assemblies from PVBA. In the following, regular arrangements formed by PVBA on $\operatorname{Ag}(111)$ in the absence of kinetic limitations are discussed. As shown in Figure 2, at small concentrations extended stripes running parallel evolve on the $\mathrm{Ag}(111)$ surface when the thermal energy is augmented by adsorption or annealing at $300 \mathrm{~K}$. Three rotational domains exist on the surface (all domains are present in the area depicted in Figure 2b), reflecting the 3-fold symmetry of the $\operatorname{Ag}(111)$ substrate. The distance between neighboring rows is on the order of $200 \AA$ in Figure 2a, the parallel alignment over this length scale being a very unusual finding. The image in Figure $2 b$ shows that small terraces are nearly empty because of migration over step edges in the ordering process.
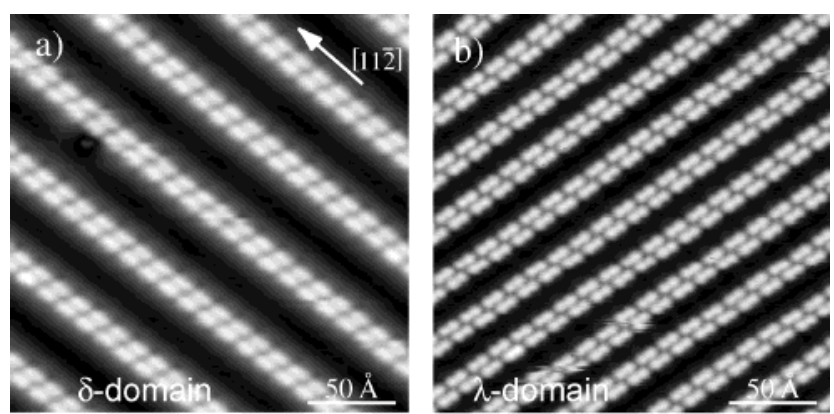

c)

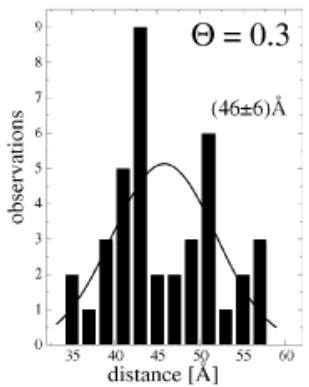

d)



e)

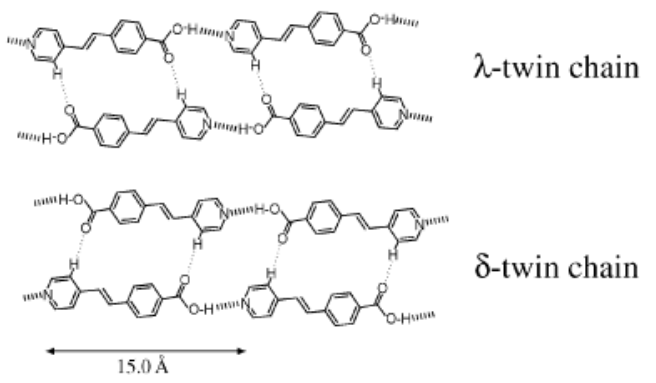

Figure 3. Formation of a regular 1-D supramolecular PVBA nanograting by H-bond mediated self-assembly on the $\mathrm{Ag}(111)$ surface at $300 \mathrm{~K}$ (following adsorption or annealing at $300 \mathrm{~K}$ ). This pattern is continued in extended domains in the micrometer range. The close-up views reveal that the stripes in Figure 2 consist of enantiomorphic PVBA twin chains. Two STM topographs illustrate the evolution with increasing coverage: (a) $\Theta_{\mathrm{ph}}$ $=0.3$ and (b) $\Theta_{\mathrm{ph}}=0.6 \mathrm{ML}$. The data similarly demonstrate the existence of different chiral domains where the supramolecular chirality of the twin chains is strictly correlated. In (c) and (d), the corresponding statistical analysis of grating periodicities is plotted. The spacing of twin chains becomes nearly equidistant at higher coverage. (e) Repeat motif of the two possible supramolecular chiral PVBA twin chains with $\mathrm{OH} \cdots \mathrm{N}$ and weak lateral $\mathrm{CH} \cdots \mathrm{OC}$ hydrogen bonds indicated.

Extended and extremely regular molecular stripe patterns evolve at higher coverages as demonstrated by the data reproduced in Figure 3. This reveals that the 1-D aggregates actually consist of PVBA twin chains, which form well-ordered nanogratings at the micrometer scale. ${ }^{23}$ The molecular axes are oriented along the chain direction, in agreement with the expected formation of hydrogen bonds between PVBA endgroups. Although atomic resolution of the close-packed Ag surface is routinely achieved with the STM employed for this study, simultaneous resolution of substrate atomic lattice and imaging of molecules proved to be impossible. In all attempts, the molecules were displaced. The chain orientation is determined by changing the tunneling parameters while recording an image and thus switching from atomic resolution to molecular imaging within one STM topograph. STM measurements performed at room temperature reveal 1-D features along the same directions. No stable tunneling conditions were obtained, and it could not be clarified whether the fuzziness of the images 

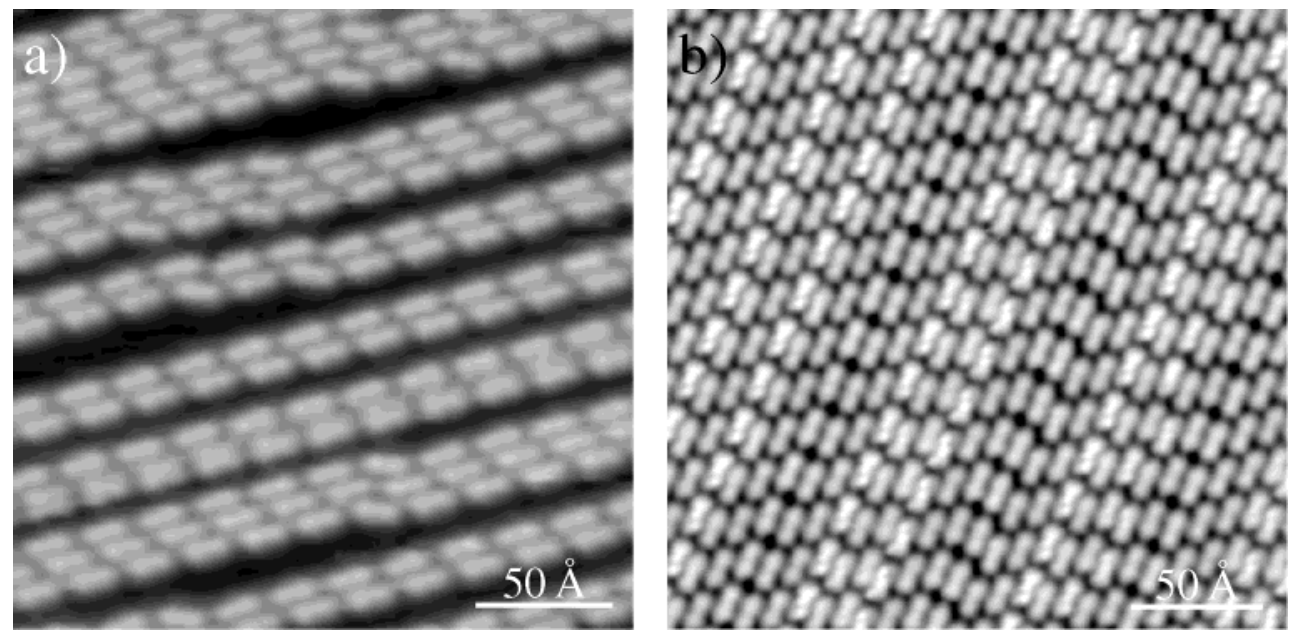

Figure 4. High coverage ordering of PVBA on $\operatorname{Ag}(111)$ (following adsorption at $300 \mathrm{~K}$ ). (a) Twin chains coexist with ribbons of three or more touching rows running parallel $\left(\Theta_{\mathrm{ph}}=0.8\right)$. Whereas the distinct shift of the twin chains is always continued in triple chains, the lateral ordering is lost in extended ribbons. (b) Monolayer structure of PVBA on $\operatorname{Ag}(111)\left(\Theta_{\mathrm{ph}}=1\right)$. The periodicity within the rows with head-to-tail coupling is maintained independently of the lateral environment. Because of space limitations, some molecular rows appearing brighter in STM images are presumably displaced vertically to reduce strain.

is because of intrinsic or tip-induced molecular motion at the surface. For the determination of the stability of the stripes, further experiments, employing, for example, scattering techniques, may be helpful.

The mutual repulsion between twin chains is reflected in the dependence on coverage of the nanogratings' periodicities. This is demonstrated by data taken for coverages of $\Theta_{\mathrm{ph}}=0.3$ and $\Theta_{\mathrm{ph}}=0.6$ in Figure 3a,b, respectively. We have performed a statistical analysis of the periodicities at different coverages. For each case, several STM images from different surface areas are evaluated, whereby the uncertainty of the determined distances falls below $1 \AA$. The statistical distribution shows that the scattering of the distances is strongly dependent on coverage. The normalized standard deviation (i.e., standard deviation divided by the mean distance) is $0.30 \pm 0.10$ at a mean twin chain distance of $56 \AA\left(\Theta_{\mathrm{ph}}=0.24\right)$ and reduces to $0.11 \pm$ 0.02 at a mean twin chain distance of $22 \AA\left(\Theta_{\mathrm{ph}}=0.6\right)$. Thus, the twin chains become nearly equidistant at higher coverage. Note, however, that the relative positioning of the molecular segments for neighboring twin chains along the chain direction is uncorrelated as the chains have a spread offset. Figure $3 \mathrm{c}, \mathrm{d}$ shows the statistical analysis corresponding to the situations in the topographs in Figure 3a,b, respectively. The interrow spacing at the highest coverage (cf. Figure $3 \mathrm{~b}$ ) falls below the length of a PVBA molecule. Nevertheless, the entire surface is exclusively covered with twin chains, and no other arrangements were found.

The twin chains resolved in Figure 3a,b are enantiomorphic, because there is a distinct directional shift in the adjacent molecular rows. For example, in the data presented in Figure $3 \mathrm{a}$, it is always the left PVBA row that appears to be displaced in the chain direction. It can be easily seen that the twin chains shown in Figure 3a cannot be transformed into those shown in Figure $3 \mathrm{~b}$ by simple translations and rotations in 2-D. Rather, a mirror operation would be required. Thus, for each rotational domain, two possibilities for supramolecular chiral ordering exist.

The twin chains run straight along a $\langle 11 \overline{2}\rangle$-direction of the Ag-lattice. This orientation is understood to be a result from the good match of the molecules' aromatic rings with high- symmetry positions of the substrate upon arrangement along this direction (for atomic modeling, cf. ref 23). The chain stride was determined to $15 \AA$, corresponding to $3 \times \sqrt{ } 3 a$ (where $a$ $=2.885 \AA$ is the $\mathrm{Ag}$ surface lattice constant), in accordance with the absence of height modulations along the chain direction. Models of the supramolecular chiral twin chains (based on an analysis of high-resolution STM data) are reproduced in Figure $3 \mathrm{~d}$ with the hydrogen bonding between the molecules indicated. ${ }^{23}$ The $\mathrm{OH} \cdots \mathrm{N}$ hydrogen bond length is estimated to be $2.5 \AA$, assuming an unrelaxed molecular geometry. For each type, only one chiral PVBA species is employed (cf. Figure 1a). Because of their antiparallel arrangement, the crooked molecules nicely match each other. Furthermore, the inward orientation of the more negative twin-bonded oxygen atoms accounts for lateral bonding and simultaneously the saturated nature of the repulsively interacting twin chains. ${ }^{24}$

A remarkable feature in the shown data is that all twin chains in the shown domains are of the same chirality (cf. Figure 3a,b); that is, there exists a mesoscopic correlation of supramolecular chirality, which was found to be strictly obeyed over the entire micrometer domain. Consequently, the racemic mixture originally deposited from the stochastic molecular beam undergoes spontaneous mesoscopic chiral resolution in the formation of the nanogratings. In a more detailed analysis of this phenomenon, metastable configurations were investigated in conjunction with MD simulations. The results suggest that the chiral resolution is related to a self-replication mechanism, which is mediated by the chiroselective attachment of molecular rows to already existing twin chains. ${ }^{24}$ It is interesting to note that the free energy of racemic gratings is expected to fall below that of the enantiopure ones, because for the long-range repulsive electrostatic interactions the chirality signature of the twin chains is irrelevant. Thus, the observed enantiopure nanogratings with their reduced entropy presumably do not represent the energetic ground state of the system.

At higher coverage, twin chains coexist with triple chains or molecular ribbons as illustrated by Figure 4a (obtained for $\Theta_{\mathrm{ph}}$ $=0.8$ ). Space limitations become important, and the energy gained by adsorption of arriving molecules overbalances the repulsive interactions between twin chains. Whereas the distinct 



Figure 5. Equilibrium structure of PEBA on $\mathrm{Ag}(111)$ (adsorbed at $150 \mathrm{~K}$, subsequently annealed to $300 \mathrm{~K}$ ). (a) Overview demonstrating the formation of 2-D islands on large terraces. Two rotational domains are discernible $\left(\Theta_{\mathrm{ph}}=0.4 \mathrm{ML}\right)$. (b) Close-up image revealing a molecular-row feature in the islands. At the atomic steps, both upper and lower edges are decorated with molecules $\left(\Theta_{\mathrm{ph}}=0.8 \mathrm{ML}\right)$

shift of twin chains is always continued in triple chains (a behavior which can also be found in triple chains evolving as a metastable species at smaller coverage and intermediate temperatures), ${ }^{24}$ this relation is generally lost when more chains come together to form extended ribbons. With increasing coverage, these ribbons grow in size, which finally leads to structures as the one depicted in Figure $4 \mathrm{~b}$ for the saturated monolayer $\left(\Theta_{\mathrm{ph}}=1\right)$. Molecules are still aligned along $\langle 11 \overline{2}\rangle$ with the periodicity in these directions strictly maintained. However, no regular 2-D superlattice is formed, as there is no unique shift between adjacent molecular rows, and the characteristic twin chain feature disappears. For some organic compounds, normally planar and rodlike molecules, there are thermodynamically stable phases in a well-defined temperature interval between the crystalline and the liquid states of aggregation. The monolayer structure of PVBA on $\operatorname{Ag}(111)$ resembles the smectic phase of such liquid crystals, where molecules are arranged in parallel layers without lateral order. However, in the smectic phase, molecules are mobile within a layer. In contrast, in the PVBA monolayer, molecules are immobile, and the structure is thus a static arrangement. Because of the lateral compression, some molecular rows are presumably slightly displaced vertically to reduce strain and thus appear brighter in STM images (cf. Figure 4b).

3.3. Two-Dimensional Hydrogen-Bonded Islands from PEBA. In the following, the self-assembly of PEBA (cf. Figure $1 b)$ is compared with the PVBA arrangements. Again, wellordered structures are formed upon annealing low-temperature grown films or adsorption at room temperature. At intermediate coverages, typically the large terraces are nearly saturated, whereas on smaller terraces, just the atomic steps are normally decorated. This signals that the molecules migrate over step edges, thus reducing the number of energetically unfavorable island boundary sites. In Figure 5a, the existence of 2-D islands at large terraces is revealed. Despite this morphological difference with respect to PVBA ordering, the chain feature is persistent within PEBA islands. This is shown by the image in Figure $5 b$, where the positioning of the individual molecules is clearly visible. The borders of the islands are almost perfectly straight (cf. Figure 5b), indicating that kinks are energetically unfavorable. The tendency to head-to-tail hydrogen bonding is also apparent from the fact that molecules at the chain ends rearrange to saturate the endgroups.

Close inspection of the images reveals that the islands represent a pair of enantiomorphic supramolecular structures because of a distinct shift of adjacent molecular chains in the islands and consequently are not simply rotational domains. A detailed data analysis reveals that the PEBA molecules do not follow a high-symmetry direction of the substrate. While the 3 -fold substrate symmetry is reflected in three rotational domains on the $\operatorname{Ag}(111)$ surface for a given enantiomorphic order, the substrate symmetry accounts for a mirror domain to every rotational domain having opposite chirality. Thus, six different domains exist. This is illustrated with two mirror-symmetric enantiomorphic domains represented in Figure 6. The molecular rows in the respective domains enclose an angle of $(94 \pm 4)^{\circ}$ and are rotated by $\pm(47 \pm 2)^{\circ}$ with respect to the [112] direction. In the case of PVBA, the mirror operation would change the chirality of each molecule. However, PEBA has an achiral molecular structure (if we disregard, as discussed, the position of the $\mathrm{H}$ atom of the $\mathrm{COOH}$ group), and the molecules in related mirror domains cannot be distinguished. In contrast to the PVBA/Ag(111) system where 2-D enantiomers assemble in supramolecular chiral structures because of resolution of a racemic mixture, in the PEBA case, enantiomorphic structures result from distinct packing of an achiral species.

A further interesting feature (cf. Figure 5b) is that both the upper and the lower substrate step edges are decorated with molecules. In contrast to low-temperature adsorption (cf. Figure 1b), steps do not act as a nucleation center. At the upper edge, double rows are aligned parallel to the monatomic step. At the lower edge, PEBA preferentially adsorbs perpendicular, and some double rows attach to these molecules. The discontinuity in the surface potential at a step results in redistribution of charge from the top to the bottom of the step. ${ }^{41,42}$ This is believed to be a reason for the affinity of weakly bound organic adsorbates to adsorb at atomic steps. ${ }^{35,43}$ Also, the reduced coordination of step atoms is frequently associated with a locally increased chemical reactivity.

(41) Smoluchowski, R. Phys. Rev. 1941, 60, 661.

(42) Avouris, P.; Lyo, I.-W.; Molinas-Mata, P. Chem. Phys. Lett. 1995, 240, 423.

(43) Kamna, M. M.; Graham, T. M.; Love, J. C.; Weiss, P. S. Surf. Sci. 1998, $419,12-23$. 


\section{substrate mirror plane I}

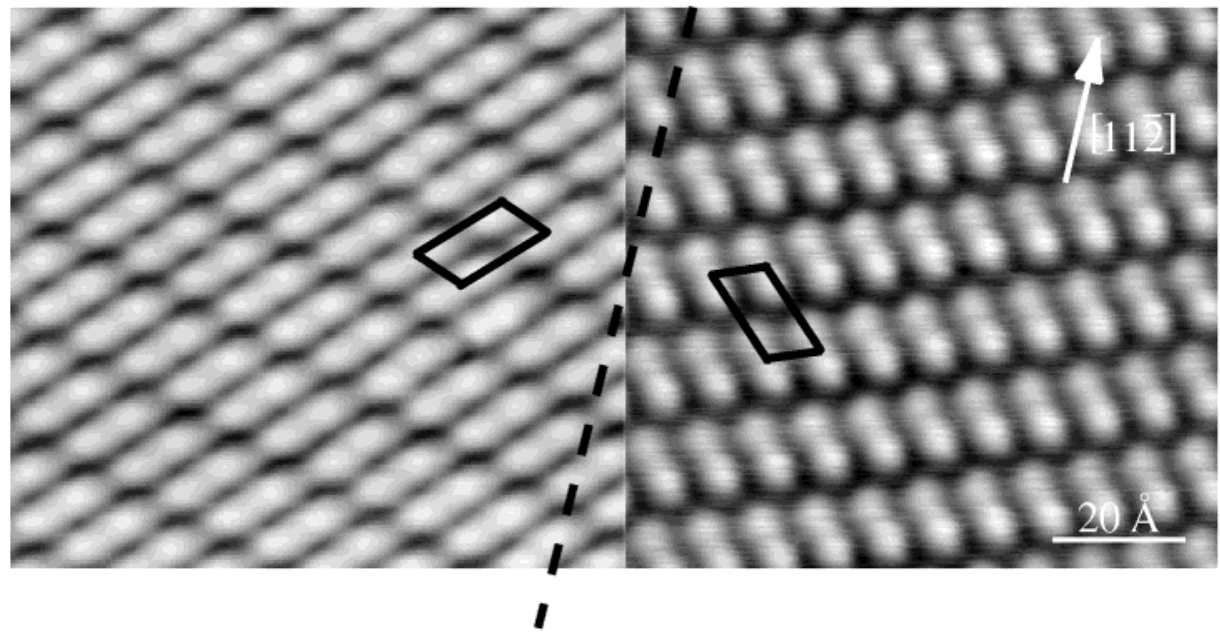

Figure 6. Enantiomorphism in PEBA islands on $\mathrm{Ag}(111)$. The distinct shift of adjacent molecular rows accounts for two mirror-symmetric species. Two mirror domains together with their respective unit cells are shown. Because of the substrate mirror symmetry along [112], there is a mirror domain with opposite chirality to every rotational domain. Note that the molecular axis is slightly rotated $\left(\sim 5^{\circ}\right)$ with respect to the chain orientation.

We determined from the STM images the substrate direction of the molecular chains in the different domains and the intermolecular distances. High-resolution data demonstrate that all molecules within the 2-D islands point in the same direction. Moreover, a close inspection of the PEBA arrangement in the islands (e.g., Figure 6) reveals that the molecular axis is slightly rotated $\left(\sim 5^{\circ}\right)$ with respect to the chain orientation. This effect can be rationalized by the directional nature of the hydrogen bond. The tilt of the molecular axis is indicated in Figure 7 where we present an $\operatorname{Ag}(111)$ atomic resolution image with the proposed model of the PEBA structure superimposed and drawn to scale. The supramolecular structure is oriented along [31 $\overline{1}$ ] (in the mirror domains along [213] $)$ with a chain periodicity of $15.3 \AA$ and an interrow distance of about $6.6 \AA$. The orientation of the molecular chains allows for the positioning of the aromatic rings close to high-symmetry positions of the substrate; that is, it is associated with the match of PEBA geometry and $\mathrm{Ag}(111)$ surface corrugation (similar to the origin of the PVBA twin chain orientation). The tilt accounts for a closer proximity and better alignment of the functional groups participating in head-to-tail and lateral noncovalent bonding.

\section{Modeling of the Supramolecular Arrangements}

4.1. PVBA Twin Chains. We modeled the molecular twin chains using force-field MD to determine the candidate stable structures, and then employed ab initio quantum calculations to validate and refine these results. In the twin chains, the molecular pair units are kept together by lateral hydrogen bonds. Thus, the bare oxygen atom in the $\mathrm{COOH}$ headgroup of each molecule has to point inward in both parallel rows, independent of their orientation (cf. Figure 8). We considered twin chains comprising both parallel and antiparallel molecular rows. Our results indicate that rows running parallel have a higher energy and therefore are less stable than the antiparallel arrangement. It is interesting to note that in the obtained parallel configuration (cf. Figure 8b), the lateral bonds are unequal (with bond lengths of 3.0 and $2.6 \AA$ ), and both differ from the two equally long lateral $\mathrm{H}$ bonds $(2.8 \AA)$ obtained for the antiparallel coupling.
This is consistent with the relative stability of the latter structure, because it is plausible that lateral bonds should be on average shorter and possibly equal to each other in the predicted stable structure. These results are in good agreement with STM observations revealing the preference of the antiparallel configuration. ${ }^{23}$ To further characterize this structure, we carried out an ab initio structural optimization of the twin chain, starting from the configuration obtained with the force-field model. The silver substrate is not taken into account in these quantum calculations, apart from imposing the constraint of planarity to the twin chain (which is reasonable for an assembly of flatlying molecules). We use periodic boundary conditions and a cubic cell with a lattice constant of $15.01 \AA$, equal to the twin chain unit length observed in the STM images. Thus, a pair of parallel molecules placed in this unit cell allows us to simulate an isolated infinite twin chain having the highest translational symmetry along the chain direction. The optimized structure obtained is reported in Figure 8a; the corresponding cohesive energy per molecule amounts to $0.434 \mathrm{eV}$. To estimate the strength of the lateral bonds between adjacent molecules, we simulate a single molecular chain using the same supercell geometry, and subtract from twice its relaxed energy value the relaxed twin chain energy. The cohesive energy associated to the overall lateral interaction obtained with this procedure is $0.090 \mathrm{eV} / \mathrm{molecule}$. This is significantly less than the $0.344 \mathrm{eV}$ computed cohesive energy per molecule in the isolated single row. Consequently, interrow bonding is weaker than intrarow bonding in these structures, which is consistent with the formation of head-to-tail coupled strings in the low-temperature aggregation. However, our results confirm that two lateral $\mathrm{H}$-bonds can form between adjacent single rows, and can be sufficiently short and strong to yield stable twin chains in the experimental temperature range.

We note that each PVBA molecule is associated to a net electric dipole, because of the overall asymmetric electric charge distribution in the molecule. On a metallic surface, long-range intermolecular interactions could be operative between adsorbates carrying electric dipoles. In the present case, however, 

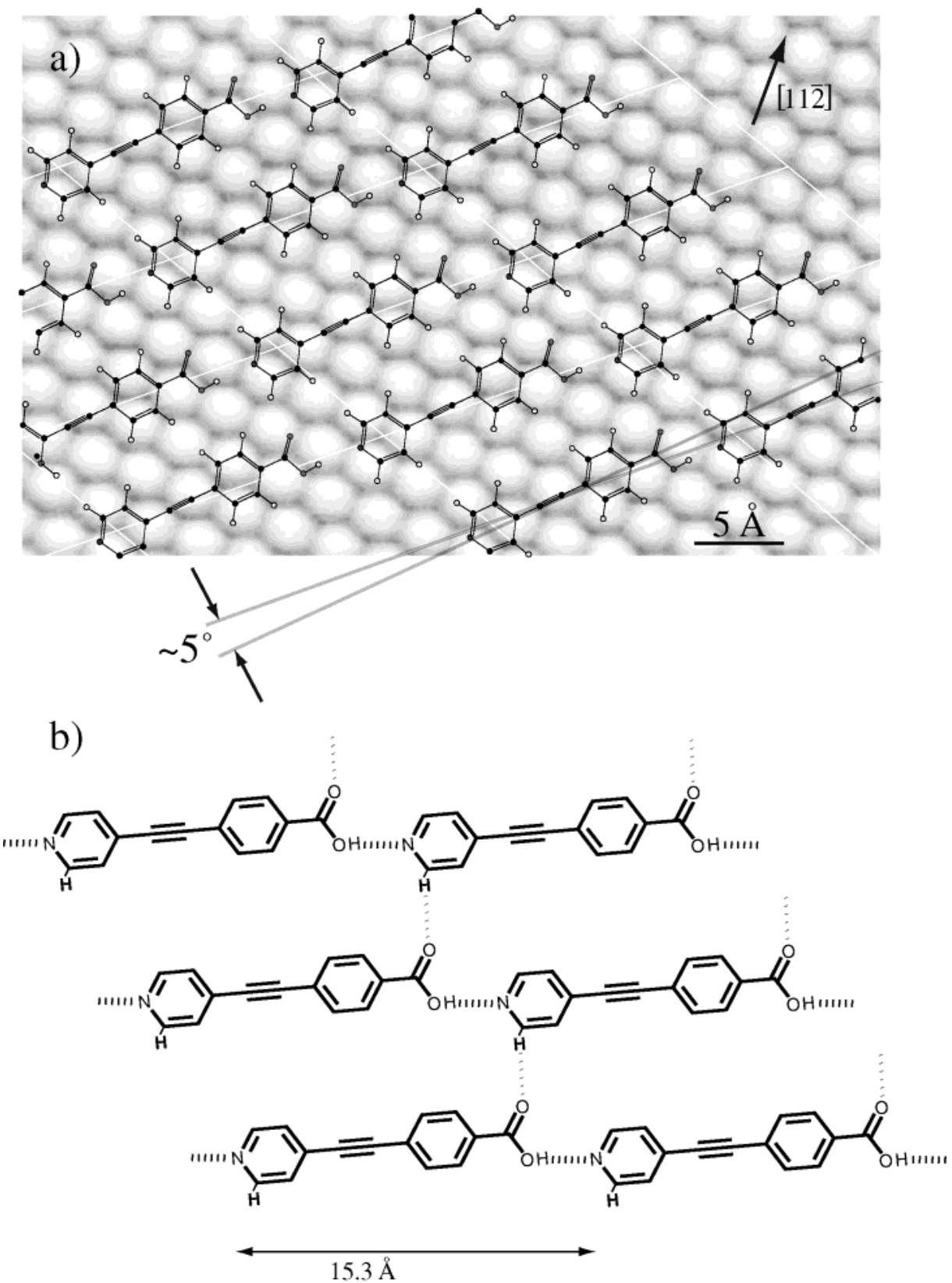

Figure 7. (a) Montage of $\operatorname{Ag}(111)$ atomic resolution image and model of PEBA layer to scale illustrating the match of molecular geometry and substrate lattice. The tilt of the molecular axis with respect to the chain orientation is indicated. (b) Repeat motif of the PEBA supramolecular structure with OH $\cdots \mathrm{N}$ and weak lateral $\mathrm{CH} \cdots \mathrm{OC}$ hydrogen bonds marked demonstrating the 2-D interconnection of the chains.

the antiparallel arrangement implies that each two-molecule unit in the PVBA twin chain carries two antiparallel dipoles. At the same time, the flat adsorption geometry implies that even the resulting quadrupole is screened by the response of the metallic surface (this is accounted for by image charges in our force model). Thus, the interaction between twin chains is expected to be negligible at long range. At close range, however, the simple electrostatic interaction picture based on multipole expansions does not hold, and the twin chains will experience a net repulsion because of the overall positive charge distribution associated to their external sides. ${ }^{24}$ Thus, the twin chains are expected to be regularly spaced in high coverage conditions, when the repulsive forces are appreciable, and more uniformly distributed at low coverage, in agreement with the experimental observations. It is interesting to note that the grating formation is thus associated with driving forces being different from those encountered in mesoscopic ordering by elastic interactions. ${ }^{4-46}$

4.2. PEBA Islands. The STM observations reported above show that the PEBA overlayer has a monoclinic structure. In our ab initio calculations, we consider a monoclinic surface cell with periodic boundary conditions in the $x y$ plane such that one PEBA molecule only is included in the repeated unit cell. The unit cell parameters are chosen to reproduce the geometry of the observed two-dimensional overlayer with packed chains running along the $[3 \overline{1} \overline{2}$ ] direction. In particular, the box length is set to the observed $15.3 \AA$ periodicity, and the interrow

(44) Barth, J. V.; Brune, H.; Behm, R. J.; Ertl, G. Phys. Rev. B 1990, 42, 93079318.

(45) Kern, K.; Niehus, H.; Schatz, A.; Zeppenfeld, P.; George, J.; Comsa, G. Phys. Rev. Lett. 1991, 67, 855-858.

(46) Vanderbilt, D. Surf. Sci. 1992, 268, L300-304. 
a)


b)
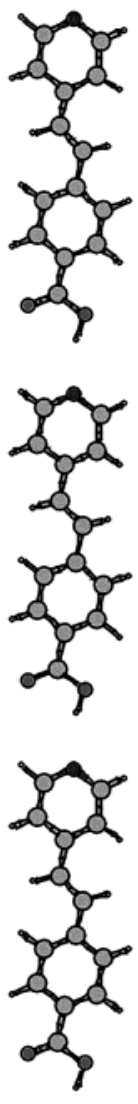
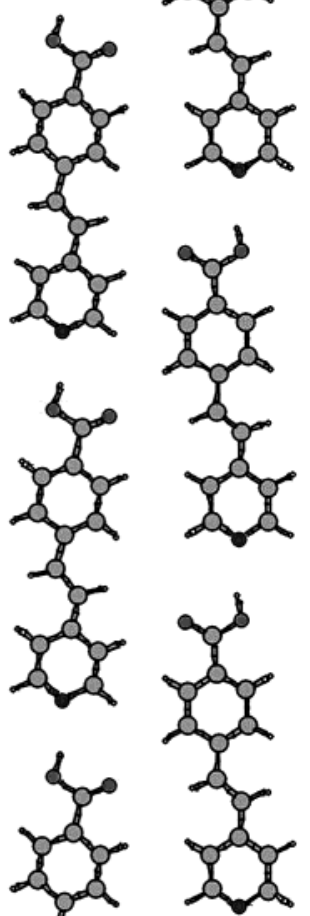

Figure 8. (a) $\mathrm{Ab}$ initio relaxed structure of a free twin chain with antiparallel orientation of the two adjacent rows. A single pair of PVBA molecules has been kept in the computational box subject to periodic boundary conditions. (b) Result of force-field simulation for twin chain with parallel orientation of the two adjacent rows. In this configuration, PVBA molecules are inequivalent.

distance is set to $6.6 \AA$. The overlayer is commensurate with the $\operatorname{Ag}(111)$ surface, yielding a $60^{\circ}$ angle between the surface cell unit vectors. Keeping this angle fixed, PEBA molecules of a given carboxylic group orientation could order in two different monolayer structures, with mirror-symmetric surface unit cells (cf. Figure 9a,b).

We find that the energetically preferred structure is the one shown in Figure 9a, which is with a cohesive energy per molecule of $0.449 \mathrm{eV}$ more stable (with a difference of 0.04 $\mathrm{eV} / \mathrm{molecule}$ ) than the alternative structure shown in Figure $9 \mathrm{~b}$, in agreement with the experimental findings (cf. Figure 6). We note that in the stable configuration the distance of the bare carboxylic oxygen to the nearest hydrogen of the adjacent molecule is smaller than in the alternative case, suggesting a stronger lateral coupling. The cohesive energy associated with the lateral coupling in the energetically preferred structure is found to be $0.109 \mathrm{eV} /$ molecule, while the cohesive energy of PEBA in an isolated single row is $0.340 \mathrm{eV} /$ molecule. Furthermore, the models predict that the molecules in PEBA islands do not run straight; they are slightly rotated clockwise with respect to the row's axis as a consequence of the formation of the $\mathrm{OH} \cdots \mathrm{N}$ chaining bond. The tilt angle between chain orientation and molecular orientation is approximately $4.6^{\circ}$, consistent with the tilt in the PEBA islands observed in the STM a)

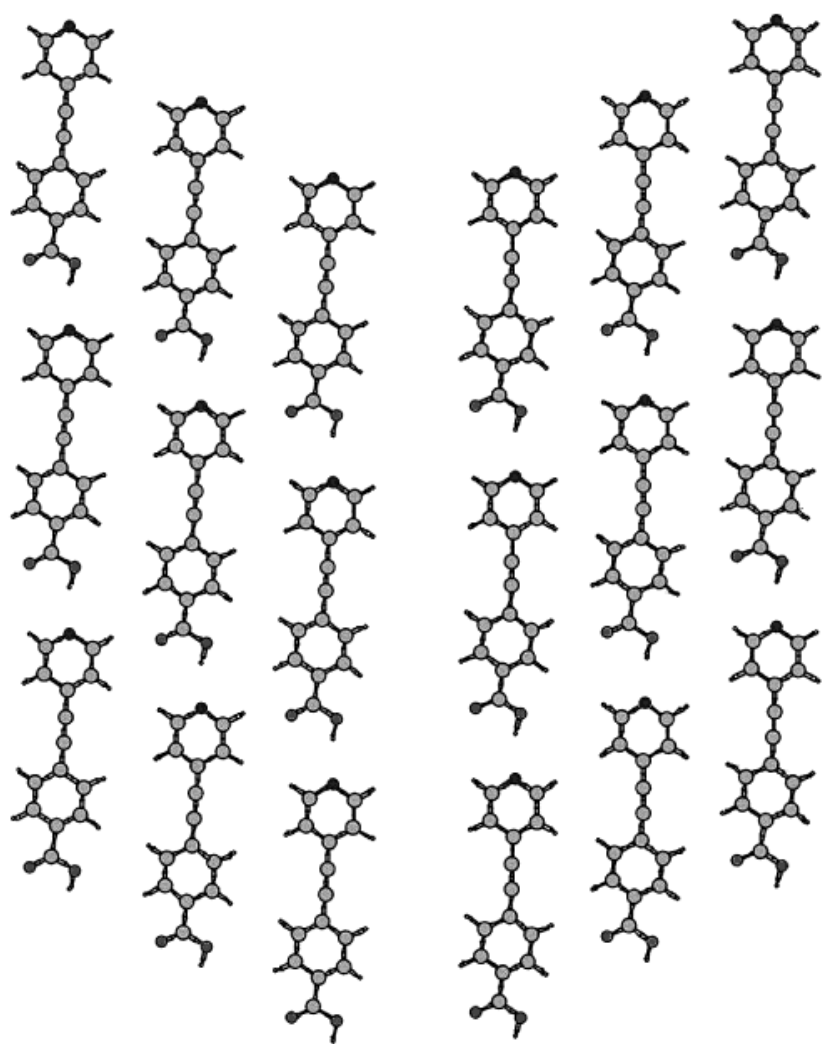

Figure 9. Simulations for the 2-D ordering of PEBA in islands. In (a) and (b) are the possible arrangements of PEBA islands. Keeping fixed the proton positioning in the carboxyl moiety and the angle of the simulation cell, we considered the two mirror-symmetric surface unit cells. (a) represents the energetically favorable configuration.

images (Figure 6). Thus, a small rotation of all molecules in a row, the same for all molecules in the 2-D arrangement, is needed to have the $\mathrm{OH}$ group pointing toward the facing nitrogen. The regular packing of PEBA molecules in ordered islands requires homogeneity of molecular rotation in neighboring rows, which in turn implies a strict correlation of the positioning of the proton in the carboxyl moiety.

\section{Comparison of the Self-Assembly Scenarios}

With the systems PEBA/Ag(111) and PVBA/Ag(111), the molecules adsorb in a flat geometry, and the balance of intermolecular and molecule-substrate interactions allows for arrangements with long-range order. The difference in the supramolecular self-assembly is the formation of 1-D twin chains in contrast with 2-D islanding. The strikingly different behavior of the two molecules can be rationalized as follows.

In both cases, the predominant intermolecular interaction is head-to-tail hydrogen bonding, which accounts for strong intrarow $(\mathrm{COOH} \cdots \mathrm{N})$ coupling and promotes directional growth. The periodic substrate corrugation and subtle differences in molecular shape and length drive the preference of PVBA for the $[11 \overline{2}]$ orientation, whereas PEBA is orientated along the [31르 direction, which is rotated by $13^{\circ}$ with respect to [11 $\overline{2}$ ]. The stride in PVBA rows amounts to $15.0 \AA$, only slightly shorter than that in the PEBA arrangements (15.3 $\AA$ ). Taking into account the molecular lengths after structural relaxation, this results in a somewhat tighter $\mathrm{OH} \cdots \mathrm{N}$ hydrogen bond 
between PVBA molecules (bond length $\approx 2.2 \AA$ ) than that between PEBA molecules (bond length $\approx 2.3 \AA$ ), which is consistent with the $0.004 \mathrm{eV} /$ molecule higher cohesive energy associated with the intrarow interaction in PVBA twin chains. Thus, the difference of $\mathrm{H}$-bond length is related to the preference for distinct orientations with respect to the Ag substrate lattice between the two molecular species. We note for comparison that in three dimensions shorter bond lengths are encountered in hydrogen-bonded crystals of nucleic acid components and isonicotinic acid (a lower analogue of PEBA), and values in the range of $1.5-2.1 \AA$ are reported. ${ }^{47,48}$

The mutual arrangement of the row features is dictated by their lateral interactions. In particular, the double-bonded carbonyl oxygen can interact electrostatically with the hydrogen atoms of adjacent molecules. PVBA and PEBA differ in length and, most importantly, in lateral shape. This implies different lateral interactions between molecules, while the assembled nanostructure grows on the surface. Adjacent rows in PVBA twin chains are aligned antiparallel and shifted. In contrast, all PEBA molecules within an island point in the same direction. In a simple dipole interaction model, the molecules should align antiparallel to minimize the electrostatic potential energy. However, this simplified picture is not applicable given the short linkage distances involved in the supramolecular assembly. Rather, the complete charge distribution with its detailed local features has to be represented to model the coupling mechanism. In the PVBA twin chains, adjacent rows are linked by two $\mathrm{CH} \cdot$ •OC hydrogen bonds per chain segment (bond distance $\approx 2.8$ A) saturating all atoms' potential hydrogen bonds. In the case of PEBA, the chains are repeatedly linked (cf. Figure 7b), and 2-D islands evolve. The interrow distance in the PEBA structure is smaller (6.6 $\AA$ ) than the one between PVBA twin chains (6.7 $\AA)$. Upon consideration of the linear molecular structure and the tilting of the molecular axis, a $0.3 \AA$ smaller $\mathrm{CH} \cdots \mathrm{OC}$ distance of $\sim 2.5 \AA$ is found for PEBA, which can again be associated with a weak hydrogen bond. ${ }^{49-51}$ Overall, the cohesive energy associated with the lateral interrow interaction is found to be $0.019 \mathrm{eV} / \mathrm{molecule}$ higher in PEBA islands.

We used MD force-field calculations to simulate molecular configurations that are not observed in the experiments, including PEBA twin chains and possible PVBA island geometries, and compared their structures and formation energies with those of the observed equilibrium structures. From the results discussed above, the requirement of forming two lateral bonds between the two molecules of each twin chain pair unit would imply a structure with antiparallel rows, analogous to the PVBA arrangement depicted in Figure 8a. However, we find that for PEBA the cohesive energy per molecule in such a twin chain

(47) Jeffrey, G. A. In Landolt-Börnstein Series VII:1b; Saenger, W., Ed.; Springer: Berlin, 1989; pp 277-348.

(48) Takusagawa, F.; Shimada, A. Acta Crystallogr. 1976, B32, 1925-1927.

(49) Desiraju, G. R. Acc. Chem. Res. 1996, 29, 441.

(50) Jeffrey, G. A. An Introduction to Hydrogen Bonding; Oxford University Press: New York, 1997.

(51) Desiraju, G. R.; Steiner, T. The Weak Hydrogen Bond; Oxford University Press: Oxford, 1999. is less than the one associated to the 2-D island structure. This is likely to be because of a less than optimal sideward bonding in the PEBA case, which is not supported here by a crooked shape of the molecule yielding smooth steric matching of antiparallel molecular pairs in the case for PVBA. To confirm this scenario, turning to the PVBA molecule, we investigated a number of candidate monolayer structures, and in all cases found a lower cohesive energy per molecule than is obtained for the PVBA twin chains. Thus, in agreement with the observations, the formation of compact islands is not favored over twin chains in the case of PVBA.

Finally, we notice that because in our model a uniform tilting angle in the PEBA rows must correspond to the same positioning of the proton in the carboxyl moiety for all molecules, the formation of antiparallel adjacent rows would yield a series of mutually repelling twin chain units, so that, as observed in the PVBA case, the twin chains would separate rather than stay packed together in a monolayer arrangement. Thus, for rows of slightly tilted PEBA molecules to form an island, they must run in a parallel orientation, consistent with the experimental observation.

\section{Conclusions}

In conclusion, we have presented a comprehensive analysis of supramolecular self-assembly and enantiomorphic ordering with two related molecular building blocks confined to a $\mathrm{Ag}$ (111) surface. Both molecules adsorb in a flat geometry. At low temperatures, they arrange in similar networks, reflecting the disposition of the molecular endgroups as active lateral bonding sites. These arrangements can be rationalized in terms of a diffusion-limited aggregation scenario of rodlike species which can relax in orientation and are subject to anisotropic interactions. In the absence of kinetic limitations, 1-D supramolecular nanogratings with tunable periodicity evolve for PVBA, which stand in striking contrast to the 2-D islanding observed for PEBA. Whereas for the PVBA/Ag(111) system chiral molecules build enantiomorphic supramolecular structures reflecting diastereoselective self-assembly and mesoscopic resolution of a 2 -D chiral species, the distinct packing of achiral molecules causes enantiomorphic structures for PEBA on the $\mathrm{Ag}(111)$ substrate lattice. Comparing the behavior of the two molecular building blocks exemplifies how subtle differences in the molecular shape drive drastic changes in 2-D selfassembly mediated by hydrogen bonding. The results reveal, in particular, that the delicate balance between moleculesubstrate and lateral intermolecular interactions responds very sensitively toward stereochemical changes in the adsorbed species. It is suggested that such effects are of general importance in the supramolecular self-assembly of nanostructures or layered materials and in the fabrication of lowdimensional supramolecular chiral systems.

Acknowledgment. We thank the Swiss National Science Foundation for financial support.

JA0256764 